\title{
A Checkpointing Algorithm Based Unreliable Non-FIFO Channels
}

\author{
Chuanqing $\mathrm{Shi}^{\mathrm{a}}$, Shengfa $\mathrm{Gao}^{\mathrm{b}}$
}

\author{
College of Computer Science and Technology, ShanDong University, Jinan Shandong
}

\begin{abstract}
We propose a coordinated checkpointing algorithm based unreliable non-FIFO channel. In unreliable non-FIFO channel, the system can lose, duplicate, or reorder messages. The processes may not compute some messages because of message losses; the processes may compute some messages twice or more because of message duplicate; the processes may not compute messages according to their sending order because of message reordering. The above-mentioned problems make processes produce incorrect computation result, consequently, prevent processes from taking consistent global checkpoints. Our algorithm assigns each message a sequence number in order to resolve above-mentioned problems. During the establishing of the checkpoint, the consistency of checkpoint can be determined by the sequence number of sending and receiving messages. We can identify the lost messages, reordering messages and duplicate messages by checking the sequence number of sending and receiving messages. We resolve above-mentioned problems by resending the lost messages, buffering the reordering messages and dropping the duplicate messages. Our algorithm makes processes take consistent global checkpoints.
\end{abstract}

Index Terms: unreliable non-FIFO channel; message losses; message duplicate; message reordering; consistent global checkpoints

(C) 2012 Published by MECS Publisher. Selection and/or peer review under responsibility of the Research Association of Modern Education and Computer Science.

\section{Introduction}

Checkpointing and rollback-recovery are popular techniques that permit processes to make progress despite a process fails. We assume that the failures are transient problems. The failures are improbable to recur when the process restarts. With this scheme, a process takes a checkpoint periodically by saving its state on stable storage [1]. When a process has a failure, it rolls back to its most recent checkpoint that saves the state of this process and restarts execution.

Most checkpointing algorithms generally assume the communication channels are reliable FIFO channels [2, 3 , 4]. Now, we propose a coordinated checkpointing algorithm based unreliable non-FIFO channel. In

The research in this paper is based on project supported by the subject of Natural Science Foundation of Shandong Province of China under grant Nos. Z2008G03.

* Corresponding author.

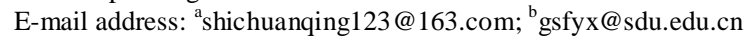


unreliable non-FIFO channel, the system can lose, duplicate, or reorder messages [5]. The processes may not compute some messages because of message losses; the processes may compute some messages twice or more because of message duplicate; the processes may not compute messages according to their sending order because of message reordering. The above-mentioned problems make processes produce incorrect computation result, consequently, prevent processes from taking consistent global checkpoints. Our algorithm can resolve these problems, and our algorithm can prevent "domino effect" and live problems associated with rollbackrecovery.

The rest of the paper is organized as follows. Section 2 develops the necessary background. In Section 3, we describe a checkpointing algorithm based unreliable non-FIFO channels. The correctness proof is provided in Section 4. Section 5 concludes the paper.

\section{Background}

\subsection{System Model}

The distributed system considered in this paper consists of $\mathrm{N}+1$ processes denoted by $\mathrm{P}_{1}, \mathrm{P}_{2}, \ldots, \mathrm{P}_{n}, \mathrm{P}_{c}$. The processes denoted by $\mathrm{P}_{1}, \mathrm{P}_{2}, \ldots, \mathrm{P}_{n}$ are ordinary processes and the process $\mathrm{P}_{c}$ is the coordinate process. The processes do not share a common memory or a common clock. Message passing is the only way for processes to communicate with each other. Each ordinary process progresses at its own speed and messages are exchanged through unreliable non-FIFO communication channel. $\mathrm{P}_{c}$ is used to coordinate the creation of the consistent checkpoints. We assume that $\mathrm{P}_{c}$ communicates with each ordinary process through reliable FIFO channel. The messages transmitted between ordinary processes are referred to as computation messages, and the messages transmitted between coordinating process and ordinary process are referred to as system messages. In order to ensure correct computation, if $\mathrm{P}_{i}$ sends computation messages to $\mathrm{P}_{j}, \mathrm{P}_{j}$ must compute the computation messages from $\mathrm{p}_{\mathrm{i}}$ according to the sending order.

Each checkpoint taken by a process is assigned a unique sequence number. The checkpoint sequence number of the process $\mathrm{P}_{i}$ is denoted by $\operatorname{csn}{ }_{i}$. The $\mathrm{j}_{t h}(\mathrm{j}>0)$ checkpoint of process $\mathrm{P}_{i}$ is assigned a sequence number $\mathrm{j}$ and $\operatorname{csn}{ }_{i}$ is set to $\mathrm{j}$. The $\mathrm{j}_{\text {th }}$ checkpoint interval[6] of process $\mathrm{P}_{i}$ denotes all the computation performed between its $\mathrm{j}_{t h}$ and $(\mathrm{j}+1)_{t h}$ checkpoint, including the $\mathrm{j}_{t h}$ checkpoint but not the $(\mathrm{j}+1)_{t h}$ checkpoint.

Each computation message sent by $\mathrm{P}_{j}$ is assigned a sequence number. The sequence number of each computation message is denoted by mid. In $\mathrm{i}_{t h}(\mathrm{i}>=0)$ checkpoint interval of $\mathrm{P}_{j}$, the mid of first computation message sent to $P_{k}(k \neq j)$ is set to 1 , and the mid of subsequent computation message sent to $P_{i}$ increases monotonically. mid of a computation message $\mathrm{m}$ is denoted by m.mid.

\subsection{Checkpoints Creation}

Chandy and Lamport [7] formally defined the concept of a consistent distributed system state. Briefly, a consistent distributed system state is formed by a set of process states. A checkpoint is a saved state of a process. A set of checkpoints, one per process in the system, is consistent if the saved states form a consistent distributed system state. 
Our algorithm saves two types of checkpoints on stable storage: tentative and permanent. A permanent checkpoint can't be undone, and a tentative checkpoint can be undone or changed to a permanent checkpoint.

Each ordinary process $\mathrm{P}_{i}$ only computes the effective computation messages in the received messages. A computation message $\mathrm{m}_{1}$ is a effective computation message if and only if $\mathrm{m}_{1}$ is first received by $\mathrm{P}_{i}$.

Definition 1. Suppose $\mathrm{P}_{1}, \mathrm{P}_{2}, \ldots, \mathrm{P}_{n}$ denote all ordinary processes in a distributed system; message sent set of $\mathrm{P}_{i}$ in $\mathrm{h}_{t h}$ checkpoint interval is defined as:

$$
\mathrm{MS}_{i}=\left\{\mathrm{MS}_{i 1}, \mathrm{MS}_{i 2}, \ldots, \mathrm{MS}_{i n}\right\}, \mathrm{i}=1,2, \ldots, \mathrm{n}
$$

Where, $\mathrm{MS}_{i j}\left(\mathrm{i} \neq \mathrm{j}\right.$ )denotes the set of the messages that $\mathrm{P}_{i}$ sends to $\mathrm{P}_{j}$ in $\mathrm{h}_{t h}$ checkpoint interval.

Definition 2. Suppose $\mathrm{P}_{1}, \mathrm{P}_{2}, \ldots, \mathrm{P}_{n}$ denote all ordinary processes in a distributed system; message received set of $\mathrm{P}_{i}$ in $\mathrm{h}_{t h}$ checkpoint interval is defined as:

$$
\mathrm{MR}_{i}=\left\{\mathrm{MR}_{i 1}, \mathrm{MR}_{i 2}, \ldots, \mathrm{MR}_{i n}\right\}, \mathrm{i}=1,2, \ldots, \mathrm{n}
$$

Where, $\mathrm{MR}_{i j}(\mathrm{i} \neq \mathrm{j})$ denotes the set of the messages that $\mathrm{P}_{i}$ receives from $\mathrm{P}_{j}$ in $\mathrm{h}_{t h}$ checkpoint interval.

We assume that $\mathrm{MR}_{i j}$. Mid denotes the maximum mid of the messages in $\mathrm{MR}_{i j}$.

Definition 3. Suppose $\mathrm{P}_{1}, \mathrm{P}_{2}, \ldots, \mathrm{P}_{n}$ denote all ordinary processes in a distributed system; message computed set of $\mathrm{P}_{i}$ in $\mathrm{h}_{t h}$ checkpoint interval is defined as:

$$
\mathrm{MC}_{i}=\left\{\mathrm{MC}_{i 1}, \mathrm{MC}_{i 2}, \ldots, \mathrm{MC}_{i n}\right\}, \mathrm{i}=1,2, \ldots, \mathrm{n}
$$

Where, $\mathrm{MC}_{i j}\left(\mathrm{i} \neq \mathrm{j}\right.$ )denotes the set of the messages that $\mathrm{P}_{i}$ computes from $\mathrm{P}_{j}$ in $\mathrm{h}_{t h}$ checkpoint interval.

Theorem 1. If $\forall \mathrm{m}_{k}, \mathrm{~m}_{k} \in \mathrm{MS}_{i j} \wedge \mathrm{m}_{k} \in \mathrm{MC}_{j i}(\mathrm{i}=1,2, \ldots, \mathrm{n} ; \mathrm{j}=1,2, \ldots, \mathrm{n} ; \mathrm{i} \neq \mathrm{j})$, then the system has a consistent distributed system state.

Proof. Since $\mathrm{MS}_{i j}(\mathrm{i} \neq \mathrm{j})$ denotes the set of the messages that $\mathrm{P}_{i}$ sends to $\mathrm{P}_{j}$ in $\mathrm{h}_{t h}$ checkpoint interval, $\mathrm{MC}_{j i}(\mathrm{i} \neq \mathrm{j})$ denotes the set of the messages that $\mathrm{P}_{j}$ computes from $\mathrm{P}_{i}$ in $\mathrm{h}_{t h}$ checkpoint interval; If $\forall \mathrm{m}_{k}, \mathrm{~m}_{k} \in \mathrm{MS}_{i j} \wedge \mathrm{m}_{k} \in \mathrm{MC}_{j i}(\mathrm{i} \neq \mathrm{j})$, which denotes that $\mathrm{P}_{j}$ has computed all the messages that $\mathrm{P}_{i}$ has sent. If $\forall \mathrm{m}_{k}, \mathrm{~m}_{k} \in \mathrm{MS}_{i j} \wedge \mathrm{m}_{k} \in \mathrm{MC}_{j i}(\mathrm{i}=1,2, \ldots, \mathrm{n} ; \mathrm{i} \neq \mathrm{j})$, which denotes that $\mathrm{P}_{j}$ has computed all the messages from other processes. If $\forall \mathrm{m}_{k}, \mathrm{~m}_{k} \in \mathrm{MS}_{i j} \wedge \mathrm{m}_{k} \in \mathrm{MC}_{j i}(\mathrm{i}=1,2, \ldots, \mathrm{n} ; \mathrm{j}=1,2, \ldots, \mathrm{n} ; \mathrm{i} \neq \mathrm{j}$ ), which denotes that all the processes has computed all the messages from other processes. In conclusion, the system has a consistent distributed system state. So the conclusion is true.

From the meaning of computer clock, the interprocess communication is not synchronous because different computer clock is difficult to achieve synchronization. The improved vector logical clock $[8,9,10]$ is proposed in this paper in order to better describe communication of inter-process. 
Definition 4. Suppose $\mathrm{P}_{1}, \mathrm{P}_{2}, \ldots, \mathrm{P}_{n}$ denote all ordinary processes in a distributed system; the improved vector logical clock of $\mathrm{P}_{i}$ is defined as:

$$
\mathrm{R}_{i}=\left(\mathrm{R}_{i 1}, \mathrm{R}_{i 2}, \ldots, \mathrm{R}_{i n}\right), \mathrm{i}=1,2, \ldots, \mathrm{n}
$$

Where, $\mathrm{R}_{i j}(\mathrm{i} \neq \mathrm{j})$ is a nonnegative integer variable maintained by $\mathrm{P}_{i}$. Its value is one larger than maximum mid of messages in $\mathrm{MC}_{i j}$.

Definition 5. Suppose $\mathrm{P}_{1}, \mathrm{P}_{2}, \ldots, \mathrm{P}_{n}$ denote all ordinary processes in a distributed system; the sending vector of $\mathrm{P}_{i}$ is defined as:

$$
\mathrm{S}_{i}=\left(\mathrm{S}_{i 1}, \mathrm{~S}_{i 2}, \ldots, \mathrm{S}_{i n}\right), \mathrm{i}=1,2, \ldots, \mathrm{n}
$$

Where, $S_{i j}(i \neq j)$ is a nonnegative integer variable maintained by $P_{i}$. Its value is equal to maximum mid of messages in $\mathrm{MS}_{i j}$.

Theorem 2. If $\mathrm{R}_{i j}=\mathrm{S}_{j i}+1(\mathrm{i} \neq \mathrm{j})$, then the messages that are sent to $\mathrm{P}_{i}$ by $\mathrm{P}_{j}$ are computed by $\mathrm{P}_{i}$.

Proof. Since the value of $\mathrm{R}_{i j}$ is one larger than maximum mid of messages in $\mathrm{MC}_{i j}$, the value of $\mathrm{S}_{j i}$ is equal to maximum mid of messages in $\mathrm{MS}_{j i}$, so the conclusion is true.

Theorem 3. If $\mathrm{R}_{i j}=\mathrm{S}_{j i}+1(\mathrm{i}=1,2, \ldots, \mathrm{n} ; \mathrm{j}=1,2, \ldots, \mathrm{n} ; \mathrm{i} \neq \mathrm{j})$, then the system has a consistent distributed system state.

Proof. Since the value of $\mathrm{R}_{i j}$ is one larger than maximum mid of messages in $\mathrm{MC}_{i j}$, the value of $\mathrm{S}_{j i}$ is equal to maximum mid of messages in $\mathrm{MS}_{j i}$. According to theorem 1 and theorem 2, so the conclusion is true.

The process of checkpointing is as follows: When $\mathrm{P}_{c}$ initiates a checkpointing process, it propagates checkpointing request to the ordinary processes. When $\mathrm{P}_{i}$ receives a checkpointing request, $\mathrm{P}_{i}$ will take a tentative checkpoint if $\mathrm{R}_{i j}=\mathrm{MR}_{i j}$. Mid $(\mathrm{j}=1,2, \ldots, \mathrm{n} ; \mathrm{i} \neq \mathrm{j})$. If $\mathrm{R}_{i j}=\mathrm{S}_{j i}+1(\mathrm{i}=1,2, \ldots, \mathrm{n} ; \mathrm{j}=1,2, \ldots, \mathrm{n} ; \mathrm{i} \neq \mathrm{j})$, we know that the tentative checkpoints are consistent according to theorem 3; so $\mathrm{P}_{c}$ informs the ordinary processes to make the tentative checkpoints permanent.

\subsection{Identification of problems}

In unreliable non-FIFO channel, the system can lose, duplicate, or reorder messages [5].

The relation of between a computation message $\mathrm{m}_{k}$ and $\mathrm{MC}_{i}$ is as follows: When $\mathrm{P}_{i}$ receives a computation message $\mathrm{m}_{k}$ from $\mathrm{P}_{j}$, if $\mathrm{m}_{k} \in \mathrm{MS}_{j i} \wedge \mathrm{m}_{k}$.mid $=\mathrm{R}_{i j}, \mathrm{P}_{i}$ will computes $\mathrm{m}_{k}$ and $\mathrm{m}_{k}$ will be put into $\mathrm{MC}_{i j}$. $\mathrm{R}_{i j}$ adds 1 automatically. If only $\exists \mathrm{m}_{l} \in \mathrm{MR}_{i j} \wedge \mathrm{m}_{l} \in \mathrm{MS}_{j i} \wedge \mathrm{m}_{l}$. mid $=\mathrm{R}_{i j}, \mathrm{P}_{i}$ will computes $\mathrm{m}_{l}$ and $\mathrm{m}_{l}$ will be put into $\mathrm{MC}_{i j}$. 
We assume that cp-state ${ }_{i}$ is a Boolean which is set to 1 if $\mathrm{P}_{i}$ is in the checkpointing process.

Definiton 6. Suppose $\mathrm{P}_{1}, \mathrm{P}_{2}, \ldots, \mathrm{P}_{n}$ denote all ordinary processes in a distributed system; If $\exists \mathrm{m}_{k},\left(\mathrm{~m}_{k} \in \mathrm{MS}_{i j} \wedge \mathrm{m}_{k} \cdot \mathrm{mid}<\mathrm{MR}_{j i} \cdot \mathrm{mid} \wedge \mathrm{m}_{k} \notin \mathrm{MR}_{j i}\right) \vee\left(\mathrm{m}_{k} \in \mathrm{MS}_{i j} \wedge\right.$ cp-state $_{i}=1 \wedge \mathrm{m}_{k} \cdot \mathrm{mid}>=$ $\mathrm{R}_{j i}$ ), which denotes message $\mathrm{m}_{k}$ is lost.

Definition 7. Suppose $\mathrm{P}_{1}, \mathrm{P}_{2}, \ldots, \mathrm{P}_{n}$ denote all ordinary processes in a distributed system; $\mathrm{P}_{j}$ receives a computation message $\mathrm{m}_{k}$ from $\mathrm{P}_{i}$.If $\mathrm{m}_{k} \in \mathrm{MS}_{i j} \wedge \mathrm{m}_{k} \cdot \mathrm{mid}>\mathrm{R}_{j i} \wedge \mathrm{m} \notin \mathrm{MR}_{j i}$, which denotes message $\mathrm{m}_{k}$ is reordered..

Definition 8. Suppose $\mathrm{P}_{1}, \mathrm{P}_{2}, \ldots, \mathrm{P}_{n}$ denote all ordinary processes in a distributed system; $\mathrm{P}_{j}$ receives a computation message $\mathrm{m}_{k}$ from $\mathrm{P}_{i}$. If $\left(\mathrm{m}_{k} \in \mathrm{MS}_{i j} \wedge \exists \mathrm{m}_{l} \in \mathrm{MR}_{i j} \wedge \mathrm{m}_{k}\right.$.mid=m $\mathrm{m}_{l}$.mid $) \vee\left(\mathrm{m}_{k} \notin \mathrm{MS}_{i j}\right)$, which denotes message $\mathrm{m}_{k}$ is duplicate.

In Fig.1, the system has taken (i-1) ${ }_{t h}(\mathrm{i}>=1)$ consistent checkpoint. $\mathrm{P}_{1}$ sends the computation message $\mathrm{m}_{1}$, $\mathrm{m}_{2}, \mathrm{~m}_{3}$ and $\mathrm{m}_{4}$ to $\mathrm{P}_{2}$ in (i-1) ${ }_{t h}$ checkpoint interval. $\mathrm{m}_{1} \cdot \mathrm{mid}, \mathrm{m}_{2} \cdot \mathrm{mid}, \mathrm{m}_{3}$. mid and $\mathrm{m}_{4}$. mid are assigned 1,2 , 3 and 4 respectively according to the sending order of messages. $\operatorname{MS}_{12}=\left\{m_{1}, m_{2}, m_{3}, m_{4}\right\}$. Message $\mathrm{m}_{1}$ first resent by $\mathrm{P}_{1}$ is denoted by $\mathrm{m}_{1}^{1}$, and message $\mathrm{m}_{1}$ resent a second time by $\mathrm{P}_{1}$ is denoted by $\mathrm{m}_{1}^{2}$.

After $\mathrm{P}_{2}$ receives message $\mathrm{m}_{3}$ and $\mathrm{m}_{4}, \mathrm{MR}{ }_{21}$ is equal to $\left\{\mathrm{m}_{3}, \mathrm{~m}_{4}\right\}$ and $\mathrm{MC}_{21}$ is NULL. $\mathrm{MR}_{21}$.mid is equal to 4. Now, $\mathrm{m}_{1}$ and $\mathrm{m}_{2}$ are in $\mathrm{MS}_{12}$, but $\mathrm{m}_{1}$. mid and $\mathrm{m}_{2}$. mid are less than $\mathrm{MR}_{21}$.mid and $\mathrm{m}_{1}$ and $\mathrm{m}_{2}$ are not in $\mathrm{MR}_{21}$; so $\mathrm{m}_{1}$ and $\mathrm{m}_{2}$ are lost during delivery and $\mathrm{P}_{2}$ will never obtain the messages.

When $\mathrm{P}_{2}$ receives message $\mathrm{m}_{3}, \mathrm{MR}_{21}$ is NULL and MC ${ }_{21}$ is NULL. $\mathrm{R}_{21}$ is equal to 1 . Now, $\mathrm{m}_{3}$ is in $\mathrm{MS}_{12}$, but $\mathrm{m}_{3}$.mid is larger than $\mathrm{R}_{21}$ and $\mathrm{m}_{3}$ is not in $\mathrm{MR}_{21}$; so $\mathrm{m}_{3}$ is reordered. When $\mathrm{P}_{2}$ receives message $\mathrm{m}_{4}$, $\mathrm{MR}_{21}$ is equal to $\left\{\mathrm{m}_{3}\right\}$ and $M C_{21}$ is NULL. $M R_{21}$.mid is equal to 3 and $\mathrm{R}_{21}$ is equal to 3 . Now, $\mathrm{m}_{4}$ is in $\mathrm{MS}_{12}$, but $\mathrm{m}_{4}$. mid is larger than $\mathrm{R}_{21}$ and $\mathrm{m}_{4}$ is not in $\mathrm{MR}_{21}$; so $\mathrm{m}_{4}$ is reordered.

After $\mathrm{P}_{2}$ receives message $\mathrm{m}_{1}^{2}, \mathrm{MR}_{21}$ is equal to $\left\{\mathrm{m}_{1}^{2}, \mathrm{~m}_{3}, \mathrm{~m}_{4}\right\}$. Because $\mathrm{m}_{1}^{2}$.mid is equal to $\mathrm{R}_{21}$, so $\mathrm{P}_{2}$ computes message $\mathrm{m}_{1}^{2}$ and $\mathrm{m}_{1}^{2}$ is put into $\mathrm{MC}_{21} . \mathrm{R}_{21}$ adds 1 automatically. After $\mathrm{P}_{2}$ receives message $\mathrm{m}_{2}^{2}$, $\mathrm{MR}_{21}$ is equal to $\left\{\mathrm{m}_{1}^{2}, \mathrm{~m}_{2}^{2}, \mathrm{~m}_{3}, \mathrm{~m}_{4}\right\}$. Because $\mathrm{m}_{2}^{2}$. mid is equal to $\mathrm{R}_{21}$, so $\mathrm{P}_{2}$ computes message $\mathrm{m}_{2}^{2}$ and $\mathrm{m}_{2}^{2}$ is put into $\mathrm{MC}_{21} \cdot \mathrm{R}_{21}$ adds 1 automatically. $\mathrm{P}_{2}$ computes message $\mathrm{m}_{3}$ and $\mathrm{m}_{3}$ is put into $\mathrm{MC}_{21}$ because $\mathrm{m}_{3}$. mid is equal to $\mathrm{R}_{21}$ and $\mathrm{m}_{3}$ is in $\mathrm{MR}_{21}$.It is the same with message $\mathrm{m}_{4}$. When $\mathrm{P}_{2}$ receives message $\mathrm{m}_{2}^{1}$, $\mathrm{MR}_{21}$ is equal to $\left\{\mathrm{m}_{1}^{2}, \mathrm{~m}_{2}^{1}, \mathrm{~m}_{2}^{2}, \mathrm{~m}_{3}, \mathrm{~m}_{4}\right\}$. Because $\mathrm{m}_{2}^{1}$. mid is equal to $\mathrm{m}_{2}^{2}$. mid, so $\mathrm{m}_{2}^{1}$ is a duplicate. When $\mathrm{P}_{2}$ receives message $\mathrm{m}_{1}^{1}$ in $\mathrm{i}_{\text {th }}$ checkpoint interval, because $\mathrm{m}_{1}^{1}$ is sent by $\mathrm{P}_{1}$ in $(\mathrm{i}-1)_{t h}$ checkpoint interval, so $\mathrm{m}_{1}^{1}$ is a duplicate.

In order to take a consistent set of checkpoints, our coordinated checkpointing algorithm must resolve message losses, message reordering and messages duplicate. The reason of livelocks [3] is that a process 
receives the same computation message twice when the process rollback recovery. We can resolve the livelocks by using the measure of resolving the messages duplicate.

\subsection{Handing the problems}

In order to get correct computation and guarantee a correct recovery following a failure, we must take a consistent set of checkpoints. So we should ensure that the above-mentioned problems are resolved correctly.

\section{Message Losses}

Message losses is defined that some messages are lost during delivery. Message losses can lead to the incorrect computation result and inconsistency. We let ordinary processes resend the lost computation messages to resolve the message losses. So we must save the determinants of each computation message on the stable storage of the sender process.

Definition 9. Suppose $\mathrm{P}_{1}, \mathrm{P}_{2}, \ldots, \mathrm{P}_{n}$ denote all ordinary processes in a distributed system; the set of sending lists is defined as:

$$
\mathrm{SQ}_{i}=\left\{\mathrm{SQ}_{i 1}, \mathrm{SQ}_{i 2}, \ldots, \mathrm{SQ}_{i n}\right\}, \mathrm{i}=1,2, \ldots, \mathrm{n}
$$

Where, $\mathrm{SQ}_{i j}$ is a list of records maintained by each process $\mathrm{P}_{i}$ for sending the computation message to $\mathrm{P}_{j}$ in $\mathrm{k}_{t h}(\mathrm{k}>=0)$ checkpoint interval. Each record has the following fields: Mid and Contents. Mid is the mid of the sent message. Contents is the contents of the sent message. $\mathrm{SQ}_{i j}[\mathrm{k}]$ is the $\mathrm{k}_{t h}$ record of $\mathrm{P}_{i}$ 's $\mathrm{SQ}_{i j}$ list;

The process $\mathrm{P}_{i}$ will save the determinants of message $\mathrm{m}_{k}$ to $\mathrm{SQ}_{i j}[\mathrm{k}]$ on the stable storage after process $\mathrm{P}_{i}$ sends a computation message $\mathrm{m}_{k}$ to $\mathrm{P}_{j}$ in $\mathrm{b}_{t h}(\mathrm{~b}>=0)$ checkpoint interval. $\mathrm{SQ}_{i j}[\mathrm{k}]$. Mid and $\mathrm{SQ}_{i j}[\mathrm{k}]$.Contents are $\mathrm{k}$ and $\mathrm{m}_{k}$ respectively. $\mathrm{P}_{j}$ will send resending message request when $\mathrm{P}_{j}$ checks that message $\mathrm{m}_{l}$ from $\mathrm{P}_{i}$ is lost. $\mathrm{P}_{i}$ receives the resending message request and resend the message $\mathrm{m}_{l}$ saved in $\mathrm{SQ}_{i j}$ to $\mathrm{P}_{j}$.

In order to make more efficient use of stable storage, each process $\mathrm{P}_{i}$ will empty $\mathrm{SQ}_{i j}(\mathrm{j}=1,2, \ldots, \mathrm{n})$ if $(\mathrm{b}+1)_{t h}$ consistent checkpoint is taken.

\section{Message Reordering}

Message reordering is defined that some messages are reordered. If we compute the messages according the receiving order, the system may lead to the incorrect result. In order to resolve the message reordering, we must let each process computes the messages from the same process according to sending order.

Definition 10. Suppose $\mathrm{P}_{1}, \mathrm{P}_{2}, \ldots, \mathrm{P}_{n}$ denote all ordinary processes in a distributed system; the set of receiving lists is defined as:

$$
\mathrm{RQ}_{i}=\left\{\mathrm{RQ}_{i 1}, \mathrm{RQ}_{i 2}, \ldots, \mathrm{RQ}_{i n}\right\}, \mathrm{i}=1,2, \ldots, \mathrm{n}
$$

Where, $\mathrm{RQ}_{i j}$ is a list of records maintained by each process $\mathrm{P}_{i}$ for saving the reordered messages from $\mathrm{P}_{j}$ in $\mathrm{k}_{\text {th }}(\mathrm{k}>=0)$ checkpoint interval, where each record has the following fields: Mid and Contents. Mid is the mid of 
the received message. Contents is the contents of the received message. $\mathrm{RQ}_{i j}[\mathrm{k}]$ is the $\mathrm{k}_{t h}$ record of $\mathrm{P}_{i}$ 's $\mathrm{RQ}_{i j}$ list;

The process $\mathrm{P}_{i}$ receives a computation message $\mathrm{m}_{k}(\mathrm{k}>1)$ from $\mathrm{P}_{j}$. If message $\mathrm{m}_{k-1}$ has not been computed, message $\mathrm{m}_{k}$ is reordered. So process $\mathrm{P}_{i}$ will save the determinants of message $\mathrm{m}_{k}$ to $\mathrm{RQ}_{i j}[\mathrm{n}](\mathrm{n}>=1)$ on the stable storage. If message $\mathrm{m}_{k-1}$ has been computed, $\mathrm{P}_{i}$ will compute message $\mathrm{m}_{k}$. If only message $\mathrm{m}_{k+1}$ is saved in $\mathrm{RQ}_{i j}[\mathrm{~m}](\mathrm{m}>=1), \mathrm{P}_{i}$ computes the message $\mathrm{m}_{k+1}$ got from $\mathrm{RQ}_{i j}[\mathrm{~m}]$ and remove the $\mathrm{RQ}_{i j}[\mathrm{~m}]$ from $\mathrm{RQ}_{i j}$.

\section{Message Duplicate}

When a process $\mathrm{P}_{i}$ receives a computation message $\mathrm{m}_{k}$ from $\mathrm{P}_{j}, \mathrm{P}_{i}$ will detect whether the message $\mathrm{m}$ is a duplicate. In our algorithm, when $\mathrm{m}$ is a duplicate message, we will drop the message.

\section{A Checkpointing Algorithm Based Unreliable Non-fifo Channels}

We suppose that the coordinate process Pc initiates the checkpointing process every a fixed time; and suppose that the checkpointing process must be finished in a fixed time. If the checkpointing process is not finished in the fixed time, the checkpoints can not be taken and the algorithm exits because of timeout.

\subsection{The Notations and The Data Structures}

The following notations and data structures are used in our algorithm:

- cp-state ${ }_{i}$ : A Boolean which is set to 1 if $\mathrm{P}_{i}$ is in the checkpointing process.

- $\operatorname{csn}_{i}$ : checkpoint sequence numbers (csn) at each process $\mathrm{P}_{i}$.

- $\operatorname{minMid}_{i}[\mathrm{j}]$ : A nonnegative integer variable maintained by $\mathrm{P}_{i}$. Its value is equal to minimum mid of messages from $\mathrm{P}_{j}$ that were saved in $\mathrm{RQ}_{i j}$ by $\mathrm{P}_{i}$.

- scount $_{i}[\mathrm{j}]$ : A nonnegative integer variable maintained by $\mathrm{P}_{i}$. Its value is equal to the number of records in $\mathrm{SQ}_{i j}$.

- rcount $_{i}$ [j]: A nonnegative integer variable maintained by $\mathrm{P}_{i}$. Its value is equal to the number of records in $\mathrm{RQ}_{i j}$.

- request: It has three fields:

$\mathrm{P}_{d}$ : the identification of a process;

Min: Its value is equal to the minimum mid of messages that should be resent;

Max: Its value is one larger than the maximum mid of messages that should be resent;

- If $\mathrm{P}_{d}=\mathrm{NULL}$, the request denotes checkpointing request, otherwise the request denotes resending message request.

- reply: It is set to 1 if ordinary processes can make the tentative checkpoints permanent; otherwise it is set to 0 if all ordinary processes should undo the tentative checkpoints and the algorithm exits because of timeout. 
cp-state $_{i}, \operatorname{csn}_{i}, \mathrm{~S}_{i j}, \operatorname{minMid}_{i}[\mathrm{j}]$, scount ${ }_{i}[\mathrm{j}]$ and rcount ${ }_{i}[\mathrm{j}]$ of $\mathrm{P}_{i}$ are initialized to $0 . \mathrm{SQ}_{i j}$ and $\mathrm{RQ}_{i j}$ of $\mathrm{P}_{i}$ are initialized to NULL. $\mathrm{R}_{i j}$ of $\mathrm{P}_{i}$ is initialized to 1 .

\subsection{Checkpointing Algorithm}

In this section, we present our blocking checkpointing algorithm.

\section{Checkpointing Initiation}

The coordinator $\mathrm{P}_{c}$ can initiate a checkpointing process. When $\mathrm{P}_{c}$ initiates a checkpointing process, it propagates checkpointing request to the ordinary processes.

\section{Reception of a request message}

A process $\mathrm{P}_{i}$ receives a request from the coordinator $\mathrm{P}_{c}$. If cp-state ${ }_{i}=0 \wedge$ request.P ${ }_{d}=\mathrm{NULL}$, the request is a checkpointing request; otherwise the request is a resending message request.

When the request is a checkpointing request, cp-state ${ }_{i}$ will be set to 1 and $\mathrm{P}_{i}$ sends $\mathrm{S}_{i}$ and $\mathrm{R}_{i}$ to coordinator $\mathrm{P}_{c}$. If $\mathrm{RQ}_{i}=\mathrm{NULL}$, which denotes that all the computation messages received by $\mathrm{P}_{i}$ has been computed, $\mathrm{P}_{i}$ will take a tentative checkpoint.

When the request is a resending message request, $\mathrm{P}_{i}$ will resend the messages whose mid is equal to or larger than request.min and less than request.max to the process request.P ${ }_{d}$.

\section{Sending a Computation Message}

When process $\mathrm{P}_{i}$ sends a computation message to process $\mathrm{P}_{j}$, it will attach the following information: mid and $\operatorname{csn}_{i}$.

\section{Receiving a Computation Message}

When process $\mathrm{P}_{i}$ receives a computation message from process $\mathrm{P}_{j}$, it will first check if rec-mid $=\mathrm{R}_{i j} \wedge$ rec$\operatorname{csn}{ }_{j}=\operatorname{csn}_{i}$. If so, $\mathrm{P}_{i}$ will compute the message and increase $\mathrm{R}_{i j}$. And then it check if the message whose mid is equal to $\mathrm{R}_{i j}$ is saved in the $\mathrm{RQ}_{i j}$ until $\mathrm{RQ}_{i}$ is NULL or the message whose mid is equal to $\mathrm{R}_{i j}$ is not saved in the $\mathrm{RQ}_{i j}$. If so, $\mathrm{P}_{i}$ gets the message from $\mathrm{RQ}_{i j}$, then $\mathrm{P}_{i}$ computes and removes the message from $\mathrm{RQ}_{i j}$. $\mathrm{P}_{i}$ increases $\mathrm{R}_{i j}$ and $\operatorname{minMid}{ }_{i}[\mathrm{j}]$ is set to the minimal mid of the messages in $\mathrm{RQ}_{i j}$. If $\mathrm{RQ}_{i j}=\mathrm{NULL}$, $\operatorname{minMid}_{i}[\mathrm{j}]$ is set to 0 ; otherwise $\operatorname{minMid}_{i}[\mathrm{j}]$ is set to the minimal mid of the messages in $\mathrm{RQ}_{i j}$.

$\mathrm{P}_{i}$ will drop the message if the message whose mid is rec-mid has been saved in the $\mathrm{RQ}_{i j}$. If rec-

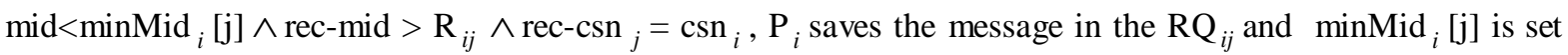
to rec-mid. $\mathrm{P}_{i}$ sends a resending request message to $\mathrm{P}_{c}$ in order to inform $\mathrm{P}_{j}$ to resend the messages whose mid 
is equal to or larger than $\mathrm{R}_{i j}$ and less than $\operatorname{minMid}_{i}[\mathrm{j}]$. If rec-mid $>\operatorname{minMid}{ }_{i}[\mathrm{j}] \wedge \operatorname{rec}-c s n_{j}=\operatorname{csn}_{i}, \mathrm{P}_{i}$ saves the message in the $\mathrm{RQ}_{i j}$.

After process $\mathrm{P}_{i}$ finishes the above actions, it will check if cp-state ${ }_{i}$ is equal to 1 . If cp-state ${ }_{i}$ is equal to 1 , $\mathrm{P}_{i}$ will take the tentative checkpoint if $\mathrm{RQ}_{i}$ is NULL.

5. Actions in the second phase for the coordinator $P_{c}$

$\mathrm{P}_{c}$ receives $\mathrm{R}_{i}$ and $\mathrm{S}_{i}$ of each process $\mathrm{P}_{i}$. If $\mathrm{R}_{i j} \neq \mathrm{S}_{j i}+1, \mathrm{P}_{c}$ will inform $\mathrm{P}_{i}$ to resend the messages whose mid is equal to or larger than $\mathrm{R}_{i j}$ and less than $\mathrm{S}_{j i}+1$. For each process $\mathrm{P}_{i}, \mathrm{P}_{c}$ will inform $\mathrm{P}_{i}$ to make its tentative checkpoint permanent if $\mathrm{R}_{i j}=\mathrm{S}_{j i}+1$. When time is timeout, $\mathrm{P}_{c}$ will inform each process $\mathrm{P}_{i}$ to cancel its tentative checkpoint.

\subsection{Algorithm Description}

Actions taken when $\mathrm{P}_{i}$ sends a computation message to $\mathrm{P}_{j}$ :

if cp-state ${ }_{i}=0$ then

$\operatorname{send}\left(\mathrm{P}_{i}, \mathrm{P}_{j}\right.$, message, mid, $\left.\operatorname{csn}_{i}\right)$;

$\mathrm{S}_{i j}:=$ mid; $\operatorname{scount}_{i}[\mathrm{j}]:=\operatorname{scount}_{i}[\mathrm{j}]+1$;

$\mathrm{SQ}_{i j}\left[\right.$ scount $\left._{j}\right]$.mid:=mid;

$\mathrm{SQ}_{i j}\left[\right.$ scount $\left._{j}\right]$.contents:=message;

Actions at process $\mathrm{P}_{i}$, on receiving a computation message from $\mathrm{P}_{j}$ :

receive $\left(\mathrm{P}_{j}, \mathrm{P}_{i}\right.$, message, rec-mid, rec-csn $\left.{ }_{j}\right)$;

if rec-mid $=\mathrm{R}_{i j} \wedge$ rec-csn ${ }_{j}=\operatorname{csn}_{i}$ then

compute the message;

$\mathrm{R}_{i j}:=\mathrm{R}_{i j}+1$;

while $\mathrm{R}_{i j}=\operatorname{minMid}_{i}[\mathrm{j}]$ do

temp:=1;

while temp $<=\operatorname{rcount}_{i}[\mathrm{j}] \wedge \mathrm{RQ}_{i j}[$ temp $] . \operatorname{mid} \neq \mathrm{R}_{i j}$ then

temp:=temp+1;

Get the message from $\mathrm{RQ}_{i j}$ [temp].

Compute the message;

Remove $\mathrm{RQ}_{i j}$ [temp] from $\mathrm{RQ}_{i j}$;

$\operatorname{rcount}_{i}[\mathrm{j}]:=\operatorname{rcount}_{i}[\mathrm{j}]-1 ; \mathrm{R}_{i j}:=\mathrm{R}_{i j}+1$;

if $\mathrm{RQ}_{i j}=\mathrm{NULL}$ then 
$\operatorname{minMid}_{i}[\mathrm{j}]=0$

else

temp:=1; $\operatorname{minMid}_{i}[\mathrm{j}]:=\mathrm{RQ}_{i j}$ [temp].mid;

while temp $<=\operatorname{rcount}_{i}[\mathrm{j}]$ then

If $\mathrm{RQ}_{i j}\left[\right.$ temp].mid $<\operatorname{minMid}_{i}[\mathrm{j}]$ then

$\operatorname{minMid}_{i}[\mathrm{j}]:=\mathrm{RQ}_{i j}$ [temp].mid;

else

Temp:=temp+1;

if rec-mid $<\operatorname{minMid}_{i}[\mathrm{j}] \wedge$ rec-mid $>\mathrm{R}_{i j} \wedge \mathrm{rec}-\operatorname{csn}_{j}=\operatorname{csn}_{i}$ then

$\operatorname{minMid}_{i}[\mathrm{j}]:=$ rec-mid; $\operatorname{rcount}_{i}[\mathrm{j}]:=\operatorname{rcount}_{i}[\mathrm{j}]+1$;

$\mathrm{RQ}_{i j}\left[\right.$ rcount $\left._{i}[\mathrm{j}]\right] \cdot \mathrm{mid}=\mathrm{rec}-\mathrm{mid} ;$

$\mathrm{RQ}_{i j}\left[\right.$ rcount $\left._{i}[\mathrm{j}]\right]$.contents $=$ message;

$\operatorname{Send}\left(\mathrm{P}_{i}, \mathrm{P}_{j}, \mathrm{R}_{i j}, \operatorname{minMid}_{i}[\mathrm{j}]\right)$;

else

if rec-mid $>\operatorname{minMid}_{i}[\mathrm{j}] \wedge$ rec-csn ${ }_{j}=\operatorname{csn}_{i}$ then

if $\mathrm{RQ}_{i j} \neq \mathrm{NULL}$ then

temp: $=1$;

While temp $<=\operatorname{rcount}_{i}[\mathrm{j}] \wedge \mathrm{RQ}_{i j}[$ temp $]$.mid $\neq$ rec-mid then

temp:=temp+1;

If temp> rcount ${ }_{i}[\mathrm{j}]$ then

$\operatorname{rcount}_{i}[\mathrm{j}]:=\operatorname{rcount}_{i}[\mathrm{j}]+1$;

$\mathrm{RQ}_{i j}\left[\operatorname{rcount}_{i}[\mathrm{j}]\right] \cdot \mathrm{mid}=\mathrm{rec}-\mathrm{mid} ;$

$\mathrm{RQ}_{i j}\left[\operatorname{rcount}_{i}[\mathrm{j}]\right]$.contents $=$ message;

else

else

Drop the message;

drop the message;

if cp-state ${ }_{i}=1$ then

if $\mathrm{RQ}_{i}=\mathrm{NULL}$ then

if $\operatorname{tckp}_{i}=1$ then

undo the tentative checkpoint;

$\operatorname{tckp}_{i}:=0$;

$\operatorname{send}\left(\mathrm{U}_{i}, \mathrm{~T}_{i}, \operatorname{mark}_{i}\right)$;

take tentative checkpoint;

$\operatorname{tckp}_{i}:=1$; 
Actions at process $\mathrm{P}_{c}$, on receiving a resending message request from $\mathrm{P}_{i}$ :

$\operatorname{receive}\left(\mathrm{P}_{i}, \mathrm{P}_{j}, \mathrm{R}_{i j}, \operatorname{minMid}_{i}[\mathrm{j}]\right)$;

request. $\mathrm{P}_{d}:=\mathrm{P}_{j}$; request.min: $=\mathrm{R}_{i j}$;

request.max: $=\operatorname{minMid}_{i}[\mathrm{j}]$;

$\operatorname{Send}\left(\mathrm{P}_{i}\right.$, request);

Actions in the first phase for the coordinate process $\mathrm{P}_{c}$ :

request. $\mathrm{P}_{d}:=$ NULL;

for $\mathrm{i}:=1$ to $\mathrm{N}$ do

$\operatorname{send}\left(\mathrm{P}_{i}\right.$, request $)$;

Actions at process $\mathrm{P}_{i}$, on receiving a request from $\mathrm{P}_{c}$ :

receive $\left(\mathrm{P}_{i}\right.$, rec-request);

if cp-state $_{i}=0 \wedge$ rec-request.P ${ }_{d}=$ NULL then

cp-state $_{i}:=1$;

$\operatorname{send}\left(\mathrm{S}_{i}, \mathrm{R}_{i}\right)$;

if $\mathrm{RQ}_{i}=\mathrm{NULL}$ then

take tentative checkpoint;

else

$\mathrm{k}:=$ rec-request.min;

while $\mathrm{k}<$ rec-request.max do

temp: $=1$;

While temp $<=$ scount $_{i}[$ rec-request. $\mathrm{Pd}] \wedge \mathrm{SQ}_{\text {irec-request. } \mathrm{Pd}}[$ temp $] \neq \mathrm{k}$ do

Temp:=temp+1;

Get the message from $\mathrm{SQ}_{\text {irec-request.Pd }}[$ temp];

$\operatorname{send}\left(\mathrm{P}_{i}\right.$, rec-request. $\mathrm{P}_{d}$, message, $\left.\mathrm{k}, \operatorname{csn}_{i}\right)$;

$\mathrm{k}:=\mathrm{k}+1$;

Actions in the second phase for the coordinate process Pc:

receive $\left(\mathrm{S}_{i}, \mathrm{R}_{i}\right)$;

num:=num+1; ack:=0;

if num $=\mathrm{N}$ then

for $\mathrm{i}:=1$ to $\mathrm{N}$ do

tag: $=0$;

for $\mathrm{j}:=1$ to $\mathrm{N}$ do

if $\mathrm{R}_{i j} \neq \mathrm{S}_{j i}+1$ then

request. $\mathrm{P}_{d}=\mathrm{P}_{j}$;

request.min: $=\mathrm{R}_{i j}$;

request.max: $=\mathrm{S}_{j i}+1$; 


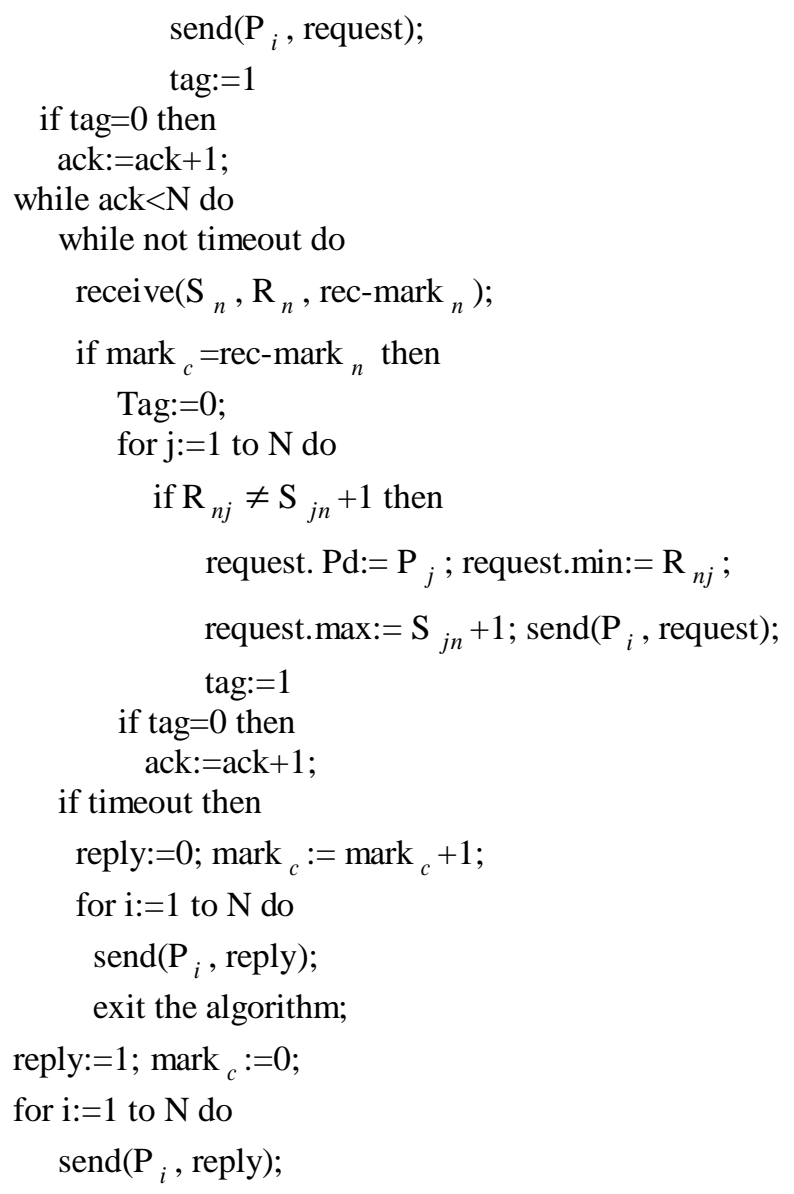

Actions at other process $\mathrm{P}_{i}$ on receiving a reply message:

receive $\left(\mathrm{P}_{i}\right.$, reply);

if reply $=0$ then

cp-state $_{i}:=0 ;$ mark $_{i}=$ mark $_{i}+1$;

else

make the tentative checkpoint permanent;

$\operatorname{mark}_{i}:=0 ; \operatorname{csn}_{i}:=\operatorname{csn}_{i}+1 ;$ cp-state ${ }_{i}:=0 ; \operatorname{tckp}_{i}:=0$;

scount $_{i}:=0 ;$ rcount $_{i}:=0 ; \mathrm{S}_{i}:=0 ; \mathrm{R}_{i}:=0 ; \mathrm{SQ}_{i}:=\mathrm{NULL}$;

\section{Algorithm Analysis}

Theorem 4. The algorithm can create consistent global checkpoints.

Proof. When $\mathrm{P}_{c}$ initiates a checkpointing process, it propagates checkpointing request to the ordinary processes. Each process $\mathrm{P}_{i}$ will send $\mathrm{S}_{i}$ and $\mathrm{R}_{i}$ to $\mathrm{P}_{c}$, and then $\mathrm{P}_{i}$ takes a tentative checkpoint if $\mathrm{RQ}_{i}$ is $\mathrm{NULL}$. 
If $\mathrm{R}_{i j}=\mathrm{S}{ }_{j i}+1(\mathrm{i}=1,2, \ldots, \mathrm{n} ; \mathrm{j}=1,2, \ldots, \mathrm{n} ; \mathrm{i} \neq \mathrm{j})$, which denotes the computation messages sent by all the sender process have been computed by their own receiver process. $\mathrm{P}_{c}$ informs each process $\mathrm{P}_{i}$ to make its tentative checkpoint permanent. Now, these checkpoints are consistent. If $\mathrm{R}_{i j} \neq \mathrm{S}_{j i}+1(\mathrm{i}=1,2, \ldots, \mathrm{n} ; \mathrm{j}=1,2, \ldots, \mathrm{n} ; \mathrm{i} \neq \mathrm{j})$, $\mathrm{P}_{c}$ will inform $\mathrm{P}_{j}$ to resend the lost messages to $\mathrm{P}_{i}$ until $\mathrm{R}_{i j}$ is equal to $\mathrm{S}_{j i}+1(\mathrm{i}=1,2, \ldots, \mathrm{n} ; \mathrm{j}=1,2, \ldots, \mathrm{n} ; \mathrm{i} \neq \mathrm{j})$. The algorithm will exit and undo the tentative checkpoints if time is timeout. In conclusion, the checkpoints created by our algorithm are consistent global checkpoints.

Theorem 5. Each process can compute the messages correctly.

Proof. Our algorithm ensures that each process computes the messages from the same process according to their sending order. When some messages are lost, the algorithm will let the sender process resend the lost messages in order that all the messages can be computed. When a process receives a duplicate message, the process don not computes the message in order that each process computes the message only once. In conclusion, each process can compute the messages correctly.

We assume that $\mathrm{n}$ is the number of processes; $\mathrm{m}$ is the number of lost messages before checkpointing phase; $\mathrm{h}$ is the number of lost messages and $\mathrm{t}$ is the number of processes that lost messages in checkpointing phase. Before checkpointing phase, process $\mathrm{P}_{i}$ checks that a computation message is lost and it inform $\mathrm{P}_{c} . \mathrm{P}_{c}$ inform the sender process to resend the lost message. In checkpointing phase, $\mathrm{P}_{c}$ sends checkpointing request to each process and each process sends a system message to $\mathrm{P}_{c}$. Eventually, $\mathrm{P}_{c}$ needs to send a reply to each process. $\mathrm{P}_{c}$ will inform the sender processes to resend the lost messages if $\mathrm{h}$ is not equal to 0 ; and then receiver processes need to send a system message to $\mathrm{P}_{c}$. So the number of system messages is $\mathrm{O}(3 \mathrm{n}+2 \mathrm{~m})$ if $\mathrm{h}$ is equal to 0 . The number of system messages is $\mathrm{O}(2 n+2 m+h)$ if $h$ is not equal to 0 .

\section{Conclusion}

In this paper, We propose a coordinated checkpointing algorithm based unreliable non-FIFO channel. In unreliable non-FIFO channel, the system can lose, duplicate, or reorder messages. The processes may not compute some messages because of message losses; the processes may compute some messages twice or more because of message duplicate; the processes may not compute messages according to their sending order because of message reordering. The above-mentioned problems make processes produce incorrect computation result, consequently, prevent processes from taking consistent global checkpoints. Our algorithm assigns each message a sequence number in order to resolve above-mentioned problems. During the establishing of the checkpoint, the consistency of checkpoint can be determined by the sequence number of sending and receiving messages. We can identify the lost messages, reordering messages and duplicate messages by checking the sequence number of sending and receiving messages. We resolve above-mentioned problems by resending the lost messages, buffering the reordering messages and dropping the duplicate messages. Our algorithm makes processes take consistent global checkpoints.

\section{References}

[1] B. Lampson and H. Sturgis, "Crash recovery in a distributed storage system," Xerox Palo Alto Research Center, Tech. Rep., Apr. 1979.

[2] Elnozahy, E. N., D. B. Johnson and W. Zwaenepoel, "The Performance of Consistent Checkpointing," Proc. 11th Symp. Reliable Distributed Systems, pp. 86-95, Oct. 1992. 
[3] Koo, R. and S. Toueg, "Checkpointing and Rollback-Recovery for Distributed Systems," IEEE Trans. Software Eng., vol. 13, no. 1, pp. 23-31, Jan. 1987.

[4] Silva, L.M. and J.G. Silva, "Global Checkpointing for Distributed Programs," Proc. 11th Symp. Reliable Distributed Systems, pp. 155-162, Oct. 1992.

[5] K. Bhatia, K. Marzullo and L. Alvisi. "The relative overhead of piggybacking in causal message logging protocols," In Proceedings of the Seventeenth Symposium on Reliable Distributed Systems, pp. 348 - 353, 1998.

[6] R. Netzer and J. Xu, "Necessary and Sufficient Conditions for Consistent Global Snapshots," IEEE Trans. Parallel and Distributed Systems, pp. 165-169, Feb. 1995.

[7] K. Chandy and L. Lamport. "Distributed snapshots: Determining global states of distributed systems,"ACM Trans. Comput. Systems, vol. 3, no. 1, pages 63-75, February 1985.

[8] Leslie Lamport. "Time, Clocks, and the Ordering of Events in a Distributed System," ACM Communications,21(7),558-565, July 1978.

[9] Mattern F. "Virtual time and global states of Distributed Systems," Proc.of Parallel and Distributed Alogrithms Conf. 215-254,1988.

[10] Shengfa Gao,Xin Li, Ruihua Zhang. "The Extended Finite State Machine and Fault Tolerant Mechanism in Distributed Systems," 2009 Seventh ACIS International Conference on Software Engineering Research, Management and Applications.33-38, 2009.

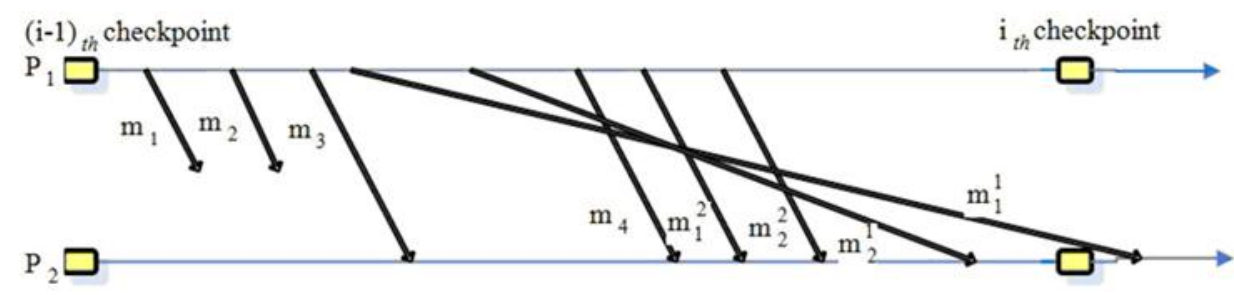

Fig. 1 